\title{
High precision and simple analog through-shaft magnetic sensors for the control of electric drives
}

Thierry Dorge, Dr.-Ing. Gerald Masson, Dr. Didier Frachon, Dr.-Ing. Stephan Biwersi, Dipl.-Ing. Michaël Del$\underline{\text { baere }}$

Moving Magnet Technologies SA, 1 Rue Christiaan Huygens, 25000 Besancon, France

Contact : info@movingmagnet.com

\begin{abstract}
This paper introduces application of a through shaft rotary magnetic position sensor technology developed by MMT to the case of large diameter shafts (typically $>50 \mathrm{~mm}$ ) which can be used to determine accurately the position of the rotor of an electric machine for smooth control and optimized efficiency.
\end{abstract}

\section{Introduction}

Many synchronous electric motors require a very accurate position sensor compatible with a sinusoidal control that will enable an efficient and smooth operation enhancing the comfort by limiting vibrations. In their basic configuration, the encoders integrated on electric motor for field oriented control are often made of a magnet in the end-of-shaft configuration. Nevertheless, in some cases, related to mechanical constraints, we have to deal with through shaft design. This feature is for example important for large power drives for electric or hybrid electric vehicles where the electric machine is located in between the combustion engine and the transmission requiring a very large through shaft design. More generally, these sensors need to keep a simple and robust design and a restricted number of parts as they are submitted to high vibration levels, a wide temperature range and speeds of several krpm.

In order to answer such requirements, MMT has developed a magnetic sensor principle which offers a competitive alternative to the conventional inductive resolver type sensors [1,2].

The basic of this solution is a through shaft angular position sensors using one or two probes of Hall effect type or GMR which measures the angle of the magnetic field generated by a magnet with $\mathrm{N}$ pole pair, that has been improved to meet very demanding accuracy requirements and able to deal with external magnetic perturbations. The number of pole pair can be equal to the number of motor pole pair in order to get an absolute position over the electric period or $\mathrm{N}$ can be equal to $1[3,4]$ in case of absolute position over $360^{\circ}$.

The paper deals with this sensor principle applied to a six pole pair machine showing that the challenge to meet less than $+/-1^{\circ}$ of electric error on a 6 pole pair magnet with inner diameter in the range of $100 \mathrm{~mm}$ was taken up.

Key features related to the robustness of the solution like effects of mechanical defaults, effects of the rotor speed and impact of stray fields on the sensor accuracy will be discussed thanks to prototype results.

\section{Multipolar through shaft standard sensor solution}

\subsection{Overall principle}

Some through shaft standard rotary position sensors rely on a sensitive device placed on an outer diameter of a multipolar magnet ring and able to measure at least two components of the magnetic field at a single point providing an absolute position inside each pole pair (Figure 1).

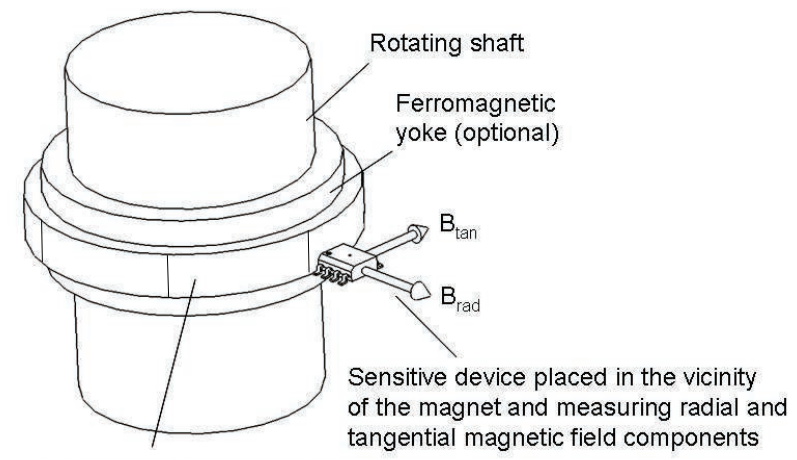

Multipolar magnetized ring permanent magnet

Figure 1: Through shaft rotary position sensor principle

In such sensors, the output signals can be either an analog sine/cosine voltage or directly an angle coming from a CORDIC algorithm or a Look-Up-Table to 
solve the arctangent calculation of the sine/cosine signals ratio.

\subsection{Case of small polar pitch magnet}

For small pole pitches a ring magnet magnetized with a classical radial magnetization has both magnetic components measured by the sensitive device close to perfect sine and cosine profiles. Unfortunately the amplitude of both components is slightly different as shown in Figure 2 what could lead to an inaccurate sensor illustrated by a non-linear variation of the magnetic angle over the mechanical stroke as shown on Figure 3 . It is well known that using a gain factor already implemented inside the Hall ASIC before making the angle calculation can lead to a significant improvement as demonstrated in Figure 3.

Figure 2 provides the example of the two magnetic field components for a small ring magnet with a shaft diameter of $16 \mathrm{~mm}$.

Figure 3 shows the theoretical field rotation and sensor non-linearity with and without gain factor showing the benefit by decreasing the electric error from $+/-9^{\circ}$ electric down to $+/-0.1^{\circ}$ electric.

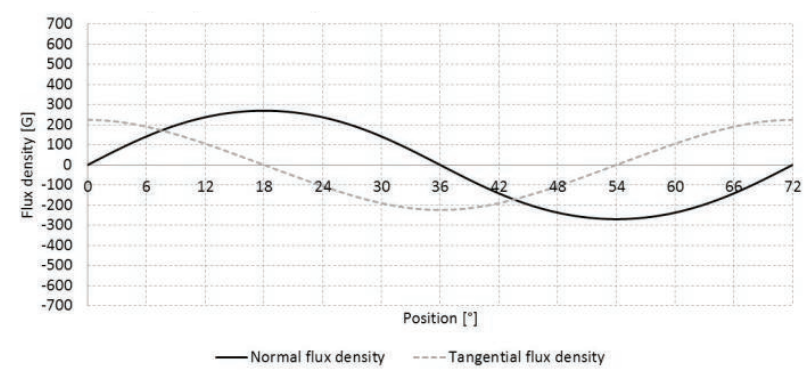

Figure 2: Magnetic field components over $72^{\circ}$ of a 5 pole pair through shaft position sensor with radial magnetization.
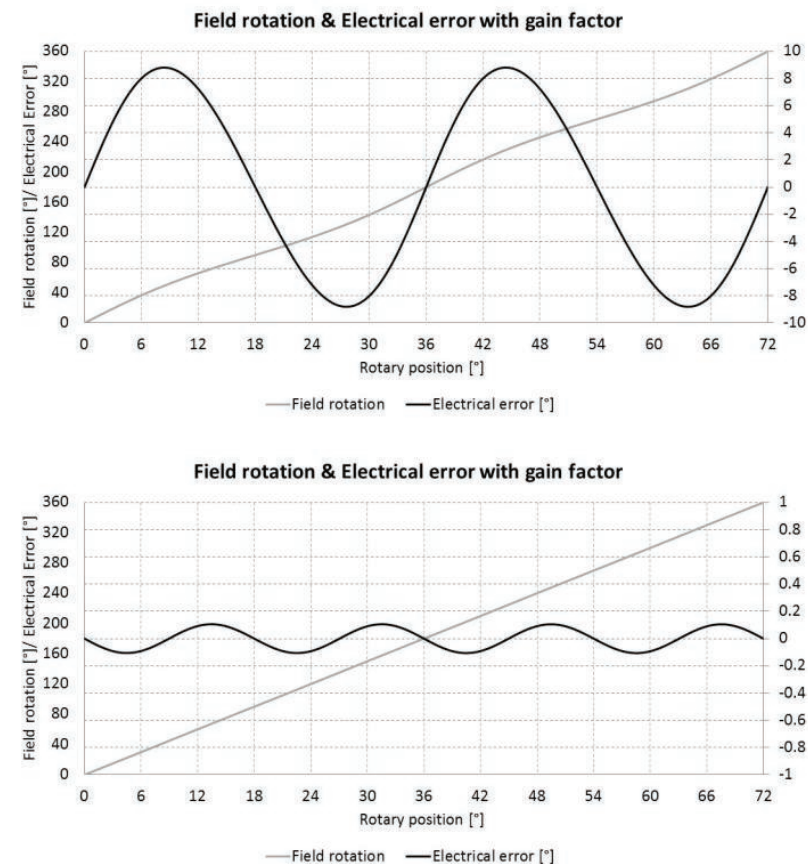

Figure 3: Non-linearity of the sensor with and without gain factor

Of course in real life, the magnet inhomogeneity, the mechanical defaults during the magnetization as well as the magnetization harmonics can lower the performances. On Figure 4, we can see the electric error measured on a 5 pole pair sensor integrated into a BLDC motor in a through shaft configuration showing a maximum error of $+/-2^{\circ}$ electric to be compared to the theoretical $+/-0.1^{\circ}$.
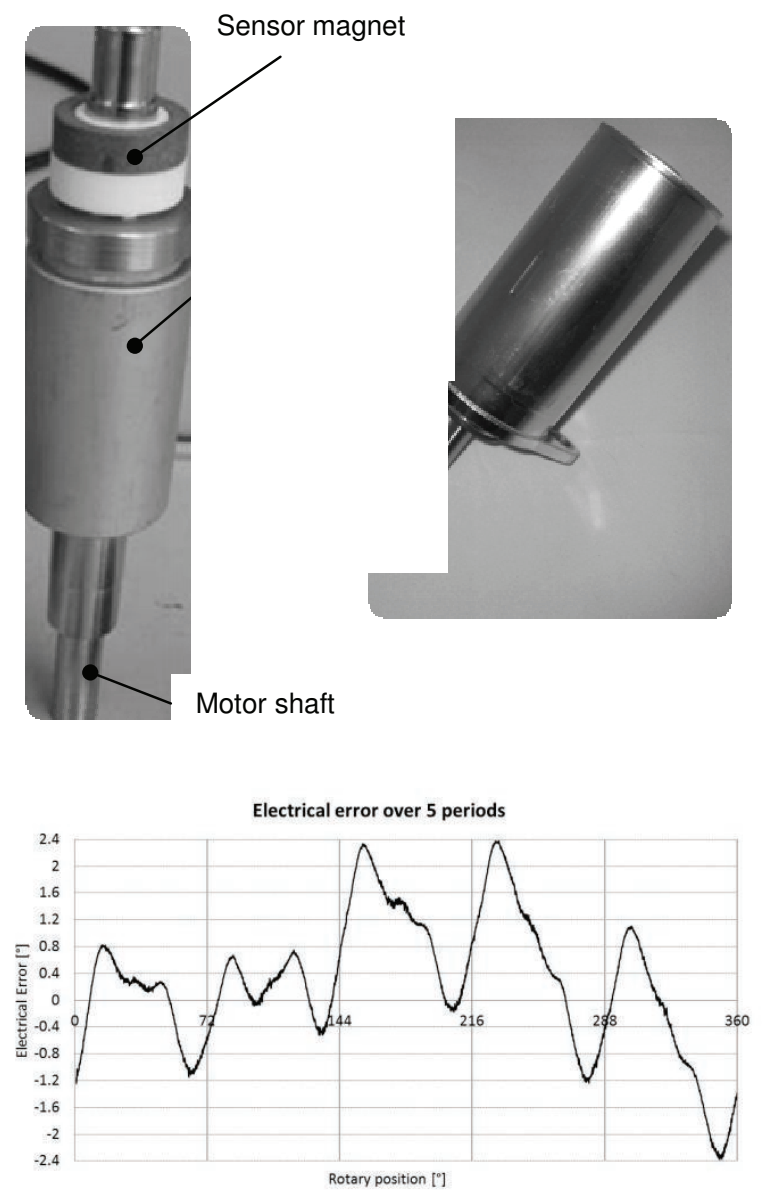

Figure 4: Prototype of the BLDC motor with its integrated analog position sensor and non-linearity measurement of the sensor with gain factor

\subsection{Case of a large diameter magnet with radial magnetization}

When the shaft diameter is getting higher with a low number of pole pair as you can find it in the machines used for the targeted automotive applications, the harmonic content of both magnetic components generated by the magnet and seen by the sensitive device is getting more and more important. In Figure 4 we have an illustration of the phenomenon on a 6 pole pair radially magnetized magnet where we can easily recognize strong $\mathrm{H} 2$ harmonic on normal component and $\mathrm{H} 3$ harmonic on tangential one. 

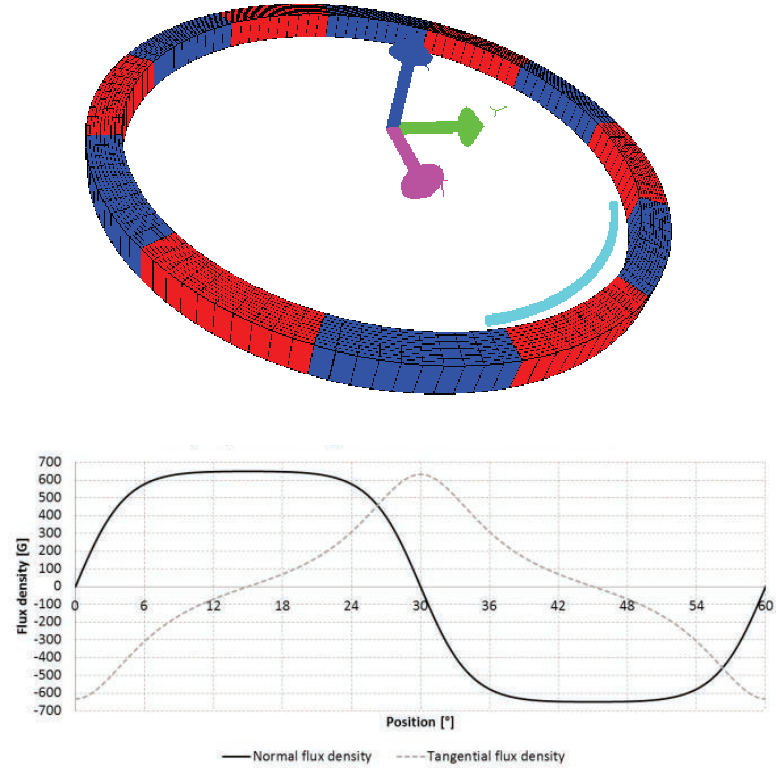

Figure 5: 6 pole pair radially magnetized magnet model (inner diameter $106 \mathrm{~mm}$ ) with inner detection and its magnetic field components over $60^{\circ}$

Such non-sinusoidal magnetic component profiles lead to a non-linear magnetic angle variation over $60^{\circ}$ and consequently a non-linear position sensor even with an appropriate gain factor as illustrated in Figure 6. Such a theoretical electric error of $+/-0.55^{\circ}$ cannot be acceptable for a smooth and efficient motor control.

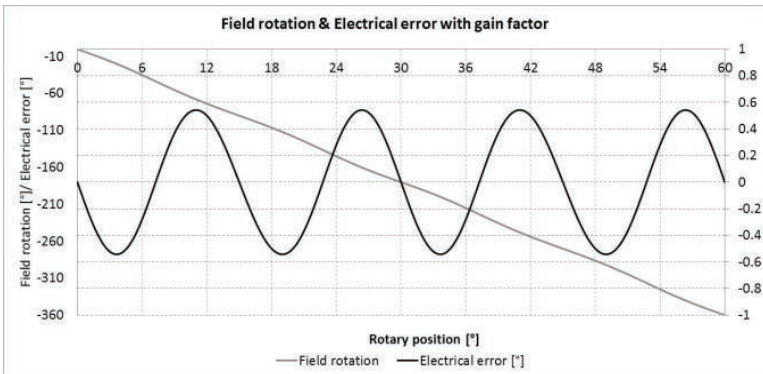

Figure 6: Non-linear magnetic angle variation and non-linearity of the rotary sensor using a radially magnetization

\section{$3 \quad$ MMT multipolar through shaft sensor solution for very high accuracy requirement}

Knowing the requirement of accuracy in the field of position sensor dedicated to motor control which is typically below $+/-1^{\circ}$ electric for large shaft diameter, MMT developed a specific sensor design using both a specific magnetization pattern and a two-probe principle.

\subsubsection{Specific magnetization}

In order to reduce the harmonic content of the signal seen by the probe, we can magnetize the magnet with a continuously variable magnetization direction as illustrated below. The main benefit is to get a very linear magnetic angle variation even for large pole pitch [5]. Of course in the scope of industrial applications this magnetization process is compatible with mass production requiring the use of standard ring magnet and reliable magnetizing fixture.

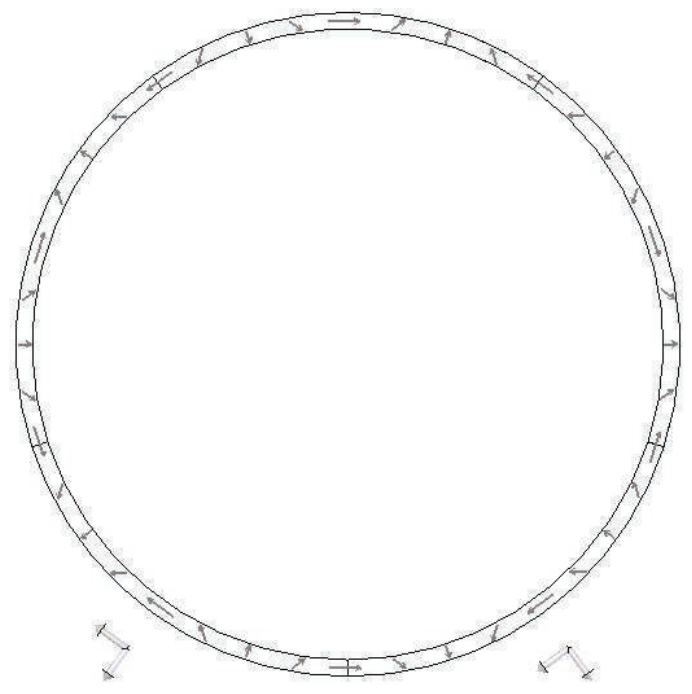

Figure 7: Example of specific magnetization pattern for a 5 pole pair magnet with inner diameter 106 $\mathrm{mm}$.

Thanks to such magnetization we can see that the theoretical error (Figure 8) is really attractive with less than $+/-0.4^{\circ}$ electric compared to the radially magnetized type (Figure 6).
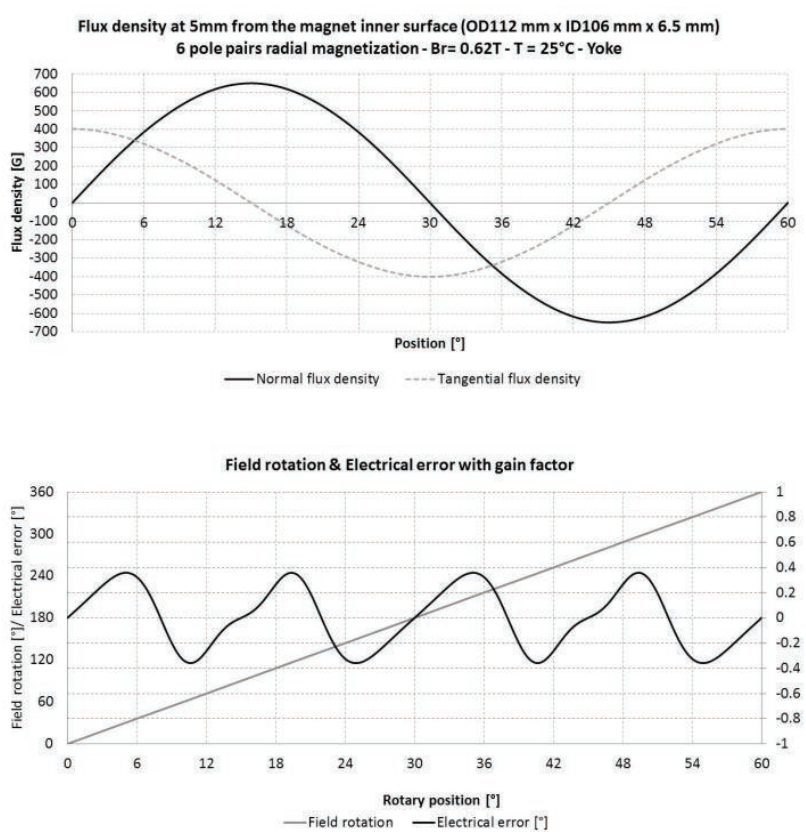

Figure 8: Both normal and tangential magnetic components generated by the 6 pole pair magnet with the specific magnetization and associated linear magnetic angle variation and electrical error. 
But as already illustrated the real life can be very different with especially the inherent residual magnetization harmonics making this sensor still accurate with its maximum $+/-1.1^{\circ}$ electric for the 6 periods measured on a prototype (Figure 9) but still not enough for the motor control purpose.

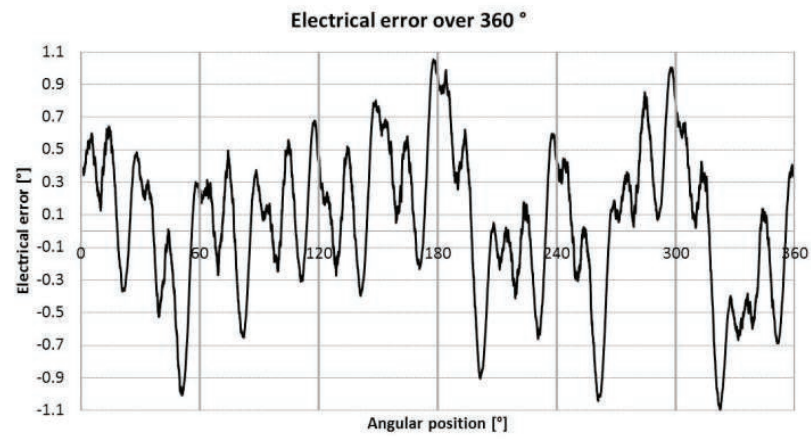

Figure 9: Measured non-linearity over $360^{\circ}$ on a 6 pole pair rotary position sensor from MMT.

\subsubsection{Two-probe principle}

As seen before, there is still a gap to fill if we want a competitive rotary sensor for motor control.

In that scope, MMT has developed a solution based on signal combination of the normal and tangential components of two Hall ICs placed at n.90 electric (Figure 10) as already described in [3,4]

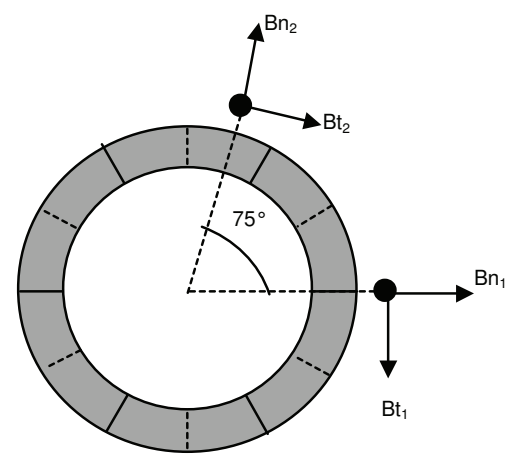

Figure 10: Principle of higher accuracy through shaft sensor applied to a 6 pole pair magnet with a second Hall probe located at $75^{\circ}$ mechanic $\left(90^{\circ}\right.$ electric) from the first one.

Using this configuration and a signal combination :

$$
\begin{aligned}
& B_{n}=f\left(B_{n 1} ; B_{t 2}\right) \\
& B_{t}=f\left(B_{t 1} ; B_{n 2}\right)
\end{aligned}
$$

it is then possible to get proper sine and cosine signals (Figure 11) having same amplitude and low harmonic content that enable to deduce (after processing) the position with a non-linearity below $+/-1^{\circ}$ electric. In Figure 12 we can see the benefit on the sensor non linearity by comparing the same period before (dotted curve) and after combination.

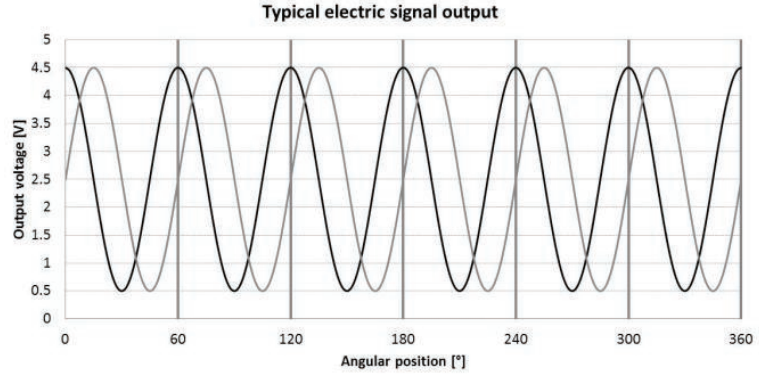

Figure 11: Measured output signals after components combination on a MMT 6 pole pair rotary position sensor.

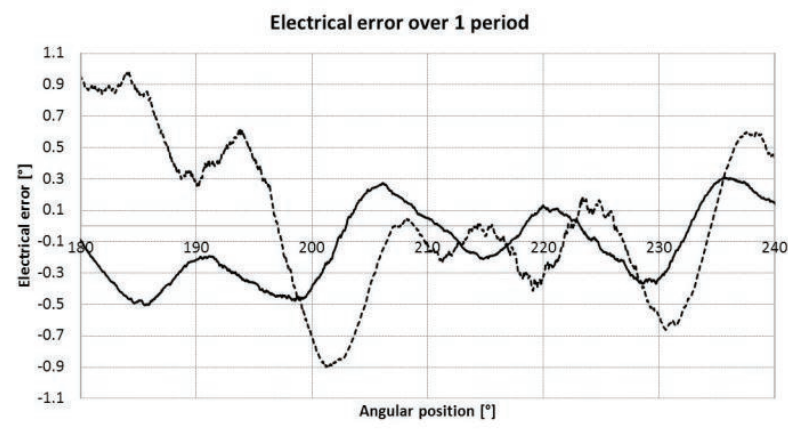

Figure 12: Measured non-linearity over a period on both MMT 6 pole pair rotary position sensor with 2-probes principle and without (dotted curve).

An other feature of this solution is to drastically reduce effects of an homogeneous external magnetic field, which can be very interesting in the case where the sensor is closely coupled to an electric motor in order to reduce the effect of the field generated by coils.

\section{Sensor robustness}

\subsection{Repeatability of the magnetization}

Of course as this sensor solution was developed for industrial applications especially for automotive field, it uses standard ring magnets (ferrite or bonded $\mathrm{NdFeB}$ as shown in Figure 13) and the magnetization process is compatible with mass production with good repeatability as illustrated in Figure 14 via the measurements of the electrical error on 3 different magnets including a current magnetization tolerance of $10 \%$ on magnet\#2. The total electrical error is below $+/-0.6^{\circ}$ on those prototypes. 


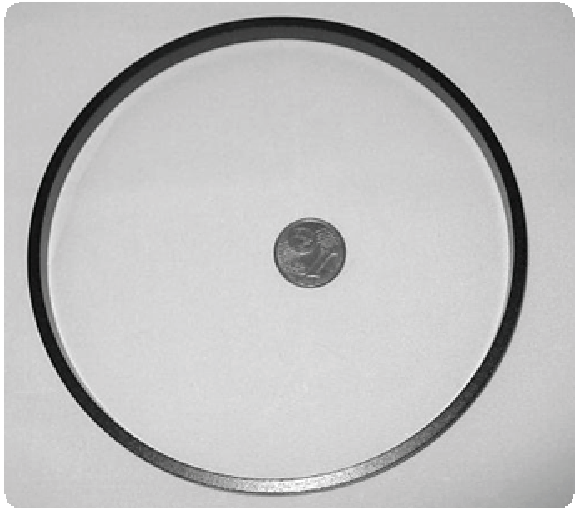

Figure 13: Bonded ring magnet with inner diameter of $106 \mathrm{~mm}$.

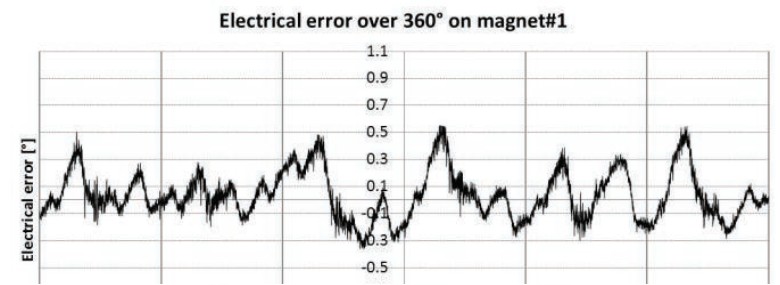

Electrical error over $360^{\circ}$ on magnet\#2

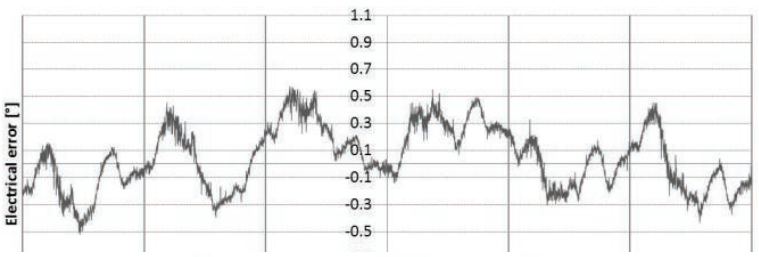

Electrical error over $360^{\circ}$ on magnet\#3

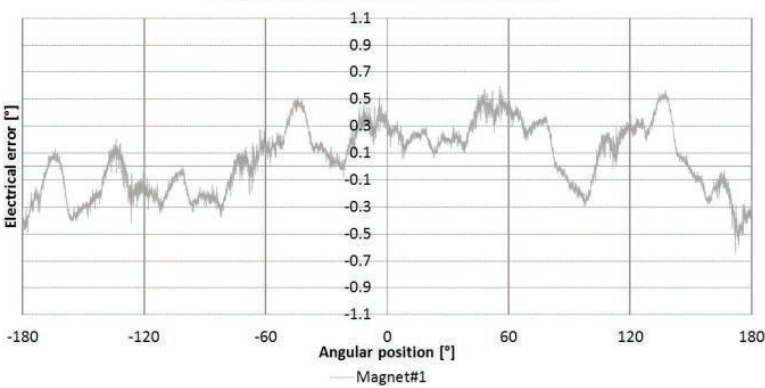

Figure 14: Sensor non-linearity over the 6 periods for 3 magnets.

\subsection{Effect of geometrical defaults}

The sensor has to deal with mechanical tolerances in production as well as in use during the lifetime of the system. Our sensor was submitted to different mechanical defaults and, the one which is illustrated below is for a $0.15 \mathrm{~mm}$ rotor off-centering in use appearing during the service. Even if we can see the footprint (dotted line) of this default through the harmonic of the $1^{\text {st }}$ order in Figure 15, the sensor initially with $a+/-0.75^{\circ}$ electric error is still accurate with its less than $+/-1.2^{\circ}$ electric error.

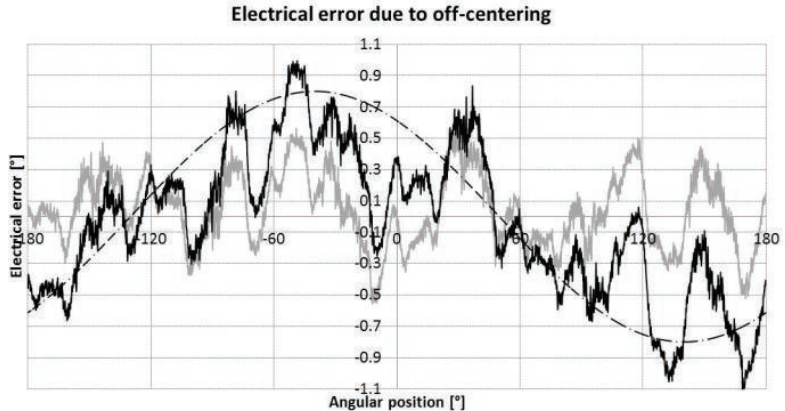

Figure 15: Impact of $0.15 \mathrm{~mm}$ rotor off-centering (black curve) on the sensor non-linearity. Grey curve is the initial curve with perfect centering.

\subsection{Effect of speed on sensor accuracy}

All the measurements shown before were done at $240 \mathrm{rpm}$ but an important point for a position sensor aimed at controlling a motor is to check the behaviour according to the motor speed. Starting with a sensor prototype which accuracy is $+/-0.82^{\circ}$ electric at $240 \mathrm{rpm}$, we can see in Figure16 the electrical error evolution which is quite constant. MMT made measurements up to $4000 \mathrm{rpm}$ and the curve from $4000 \mathrm{rpm}$ up to $12 \mathrm{krpm}$ is a projection based on the model.

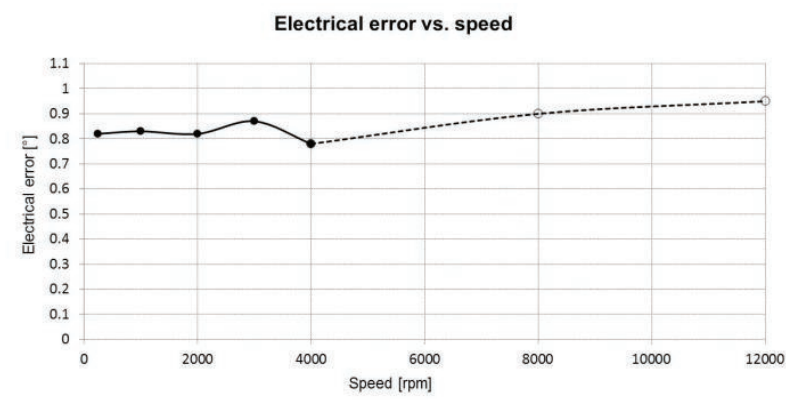

Figure 16: Effect of rotor speed on the sensor accuracy.

The 2-probe principle is able to balance the drift, intrinsic to the Hall probe, which appears between one electric component and the other one. Nevertheless, even if speed has no impact on the sensor nonlinearity as shown, we have to notice a phase lag phenomenon intrinsic to the Hall probe which increases linearly with the speed corresponding to an electric signals sliding versus the mechanical position. This one could be anticipated on the motor microcontroller but MMT is also investigating the benefit which one can get from GMR probe type.

\subsection{Effect of an external magnetic field}

One of the feature of the signal combination solution described here above is to theoretically provide a direct cancellation of an homogeneous external field, keeping in mind however that in reality this field may be more or less homogeneous.

Figure 17 shows results obtained with homogeneous stray fields of 25 and 50 Gauss directly applied on the magnet and probes. It is 
important to notice that no additional shieldings made thanks to magnet yoke or whatever are integrated.

We can notice that the overall linearity of the sensor stays however in the range of $+/-1^{\circ}$ electric.

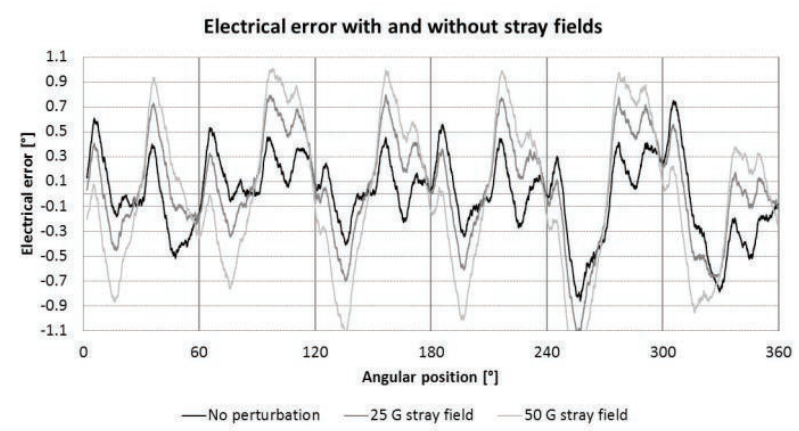

Figure 17: Effect of stray fields on electrical error.

Before the final integratation of the sensor on the 6 pole pair machine MMT is making tests with non homogeneous stray fields coming from an electrical coil by taking into account also the outer magnet yoke which should bring an intrinsic shielding effect.

\section{Conclusions and outlooks}

In the scope of synchronous electric motor control, there is a requirement for a multipolar sensor able to ensure the motor efficiency and dynamic torque smoothness thanks to its high accuracy. When diameters are getting to increase, the standard approach based on radially magnetized magnet is no more able to meet the requirements.

In this paper, MMT has proposed a solution to deal with through shaft position sensor made of a multipolar magnet for large diameters able to provide accuracies in the range of $+/-1^{\circ}$ electric as requested by electric motor engineers. This was achieved thanks to a specific magnetization pattern and a 2-probe principle as shown with the help of prototype measurements. It was also important to focus on the robustness point of view which can make this development a competitive alternative to the multipolar "resolver" and for that we analyzed the influences of mechanical defaults, stray fields, and speed as well as magnetization repeatability.

The next step for this sensor development is now to be integrated on the electric machine and to use it for field oriented control.

\section{Literature}

[1] http://en.wikipedia.org/wiki/Resolver_(electrical)

[2] Kitazawa, K., Principle and application of resolvers for hybrid electric vehicles, Proc. of Innovative Automotive Transmissions Conference 2009
[3] "Angular or linear magnetic position sensor not sensitive to external fields", PCT application WO09101270 under french priority FR2923903 granted on 8/01/2010.

[4] "Absolute magnetic sensors for large diameter through-shaft applications", Sensoren und Messesysteme 2010, Nürnberg, D. Frachon, G. Masson, T. Dorge, M. Delbaere, S. Biwersi.

[5] "Position sensor with variable direction of magnetization and method of production", EP1989505 granted on 21/07/2010. 